Témoigner Témoigner. Entre histoire et mémoire

Getuigen Revue pluridisciplinaire de la Fondation Auschwitz

$127 \mid 2018$

Perpétuation de la violence après 1918

\title{
Lettres des camps de concentration nationaux-socialistes et contribution de celles-ci à la recherche historique
}

Jean-Louis Rouhart

\section{OpenEdition}

Journals

Édition électronique

URL : https://journals.openedition.org/temoigner/7838

DOI : 10.4000/temoigner.7838

ISSN : 2506-6390

Éditeur :

Éditions du Centre d'études et de documentation Mémoire d'Auschwitz, Éditions Kimé

Édition imprimée

Date de publication : 1 octobre 2018

Pagination : 100-109

ISBN : 978-2-930953-076

ISSN : 2031-4183

Référence électronique

Jean-Louis Rouhart, "Lettres des camps de concentration nationaux-socialistes et contribution

de celles-ci à la recherche historique », Témoigner. Entre histoire et mémoire [En ligne], 127 | 2018, mis en ligne le 10 février 2022, consulté le 14 février 2022. URL : http://journals.openedition.org/ temoigner/7838; DOI : https://doi.org/10.4000/temoigner.7838 


\section{Lettres des camps de concentration nationaux-socialistes et contribution de celles-ci à la recherche historique}

Cette étude, intitulée Beitrag der illegalen Korrespondenz als Forschungsquelle zur Geschichte der deutschen NS-Konzentrationslager. Pädagogische Anwendungsmöglichkeiten (manuscrit) s'est vue attribuer en juin 2011 le Prix « Fondation AuschwitzJacques Rozenberg ». Une version abrégée en langue française a été publiée en 2015 par les Éditions des Territoires de la Mémoire sous le titre Lettres de l'ombre. Correspondance illégale dans les camps de concentration nazist.

Pour réaliser notre étude, nous nous sommes penchés sur des lettres conservées aux archives des différents mémoriaux des camps de concentration, aux archives de l'United States Holocaust Memorial Museum ainsi que sur des extraits de lettres publiés dans différents ouvrages et revues. Ces sources n’avaient jamais fait jusque là l’objet d'une analyse globale et approfondie. Elles avaient certes déjà été étudiées d'un point de vue philatélique, sollicitées pour leurs informations historiques, utilisées en tant que preuves juridiques et insérées dans la problématique générale de la Résistance dans les camps. Cependant, ces lettres n’avaient jamais été analysées été répertoriées en fhématique, stylistique ou littéraire. Elles n’avaient jamais été répertoriées en fonction de leurs particularités ni étudiées sur la base de leurs fonctionnalités. Les caractéristiques de leurs auteurs, leur statut épistémologique, leurs potentialités pédagogiques n’avaient jamais été traités, alors que paradoxalement on constatait - et constate encore - un intérêt pour les témoignages oraux qu'elles ont laissée.

Dans notre étude, nous nous sommes d'abord employés à préciser dans une introduction les notions de lettres w, "camps deconcentration nationaux-socialistes et «illégales » afin de déterminer quels documents et quels camps pouvaient faire l'objet de notre analyse. Ainsi, sous le terme «lettres », entendu au sens large, nous avons pris en considération toutes sortes d'écrits épistolaires, y compris les billets dissimulés dans divers objets, les bouts de papier jetés des «Trains de la Mort » et les messages enfouis près des fours crématoires. Nous avons exclu toutefois les écrits de propagande distribués aux alentours des camps, les photographies prises clandestinement et les journaux intimes rédigés sous forme de lettres; par ailleurs, seules les lettres illéges sortant des camps ontété soumises à notre alyse, étant deules les étant donné que gếraén detruites pour des rasen lement les lettres provenant des camps (principaux et annexes) soumis successivement à l'Inspection SS des camps de concentration (IKL), puis à l'Office central SS pour l'économie et l'Administration (WVHA), mais aussi les lettres jetées des trains transportant les détenus vers les camps (du fait que ces trains faisaient pratiquement partie de l'espace concentrationnaire). Ont fait également l'objet de notre analyse les lettres issues des centres d'extermination, celles émanant des camps de « transit » et d'« internement » ainsi que les lettres du « ghetto » de Terezín, laissant ainsi de côté la correspondance en provenance des autres lieux de détention nazis tels cue les camps de travaux forcés ou les « camps d'éducation par le travail ».
En examinant les documents officiels nazis tels que les ordres d'Hitler, les décrets de l'Office central de la sécurité du Reich (RSHA) et de l'Office central SS pour l'économie et l'administration (SS-WVHA), et d'autres ordonnances encore, nous avons pu établir les catégories de concentrationnaires qui étaient exclues de tout trafic postal, celles qui étaient défavorisées ou privilégiées en matière de correspondance et dans quels cas des lettres de camps peuvent être considérées, en raison de leurs caractéristiques formelles ou thématiques, comme illégales. À ces fins, nous avons également examiné et comparéles points les plus importants des extraits des règlements des camps relatifs au courrier postal et décrit les conséquences concrètes, essentiellement d'ordre répressif, que le non-respect des dispositions réglementaires entraînait pou 
familles, qu'il y avait eu de nombreuses interdictions temporaires et qu'en raison des contrôles stricts des bureaux de censure le contenu des lettres officielles s'étai limité généralement à des banalités se rapportant à la soi-disant bonne santé de détenus et à des demandes de colis.

Plus loin dans létude, nous nous sommes interrogés sur l'ampleur du phénomène des lettres illégales. En nous basant sur les décrets officiels, les communication et ordonnances de la SS ainsi que sur les récits des témoins oculaires, nous avons et ord nous avon évoqué le fait que les documents, dont nous disposons aujourd hui, ne sont vraisemére entre les détenus et le monde extérieur, malgré la surveillance étroite dont elle faisait l'objet.

Après quoi, nous avons examinéle sens et la finalité du courrier illégal des camps, en montrant l'importance que revetait cette correspondance pour les prisonnier et les différentes fonctions qu'elle remplissait dans les camps. C'est ainsi que nous avons relevé des aspects communicatifs (établissement d’un véritable dialogue avec les proches, libre expression de sentiments, interruption de l'isolement), informatifs (révélation des crimes nazis, fourniture de données pour des brochures et écrits de propagande, preuves pour la condamnation des criminels), consiratifs te (contacts avec les on vanisations de la Résistance àlextérieur des camps en vue la préparation dévasions, de sabotages et de révoltes, organisation de fraudes de médicaments, de denrées alimentaires et darmes). En outre, nous avons mis en évidence des aspects thérapeutiques (rétablissement de la liaison avec le monde extérieur, amélioration de la capacité de résistance morale, libération mentale de la tyrannie nazie), cognitifs (meilleure compréhension des événements et de la propre personnalité) ainsi que des fonctions d'ordre psychologique (élimination des agressions), transcendantal (fuite intellectuelle), philosophique (réflexion sur le sens de la vie), moral (renforcement d'anciennes convictions ou découverte de nouvelles valeurs) et humaniste (affirmation de soi, rétablissement des droits individuels et de la dignité humaine).

Nous nous sommes également penchés sur les caractéristiques formelles et thématiques des différentes catégories de lettres illégales et à côté des lettres illustrées (transmises par des prisonniers jouissant de privilèges et exprimant leur profonde nostalgie du monde vécu avant la déportation), des lettres rédigées en langues étrangères (émanant également de prisonniers privilégiés) et des lettres transmises par des expéditeurs faux ou fictifs (privés de l'autorisation d'écrire), nous avons porté notre attention sur les lettres porteuses de messages secrets, les lettres clandestines (les Kassiber), les lettres jetées des «Trains de la Mort», ainsi que les lettres enfouies dans le sol à l'intérieur des camps.

En ce qui concerne les lettres recélant des messages secrets, nous avons décri dans notre étude les techniques utilisées pour dissimuler ces messages. Celles-ci s'avèrent être, pour la plupart, d’ordre stéganographique. Nous avons ainsi mentionné le recours à l'urine utilisée comme encre sympathique (notamment par les « cobayes » du camp de Ravensbrück afin de dénoncer les expériences médicales qui étaient pratiquées), l'emploi de mots étrangers hébreux, basques ou romanis, inconnus des censeurs, et de mots codés tels que des noms de famille évoquant les dirigeants des Alliés. Nous avons cité également quelques exemples d’allusions comprises par les seuls destinataires et révélé l'existence de systèmes sophistiqués de codes chiffrés ou basés sur certaines lettres, et même sur certains jambages²

Lors de l'examen du groupe de lettres illégales le plus important, celui des envois clandestins, nous avons tenté de cerner l'identité et la personnalité des intermédiaires («passeurs »), de reconstituer l'importance du trafic postal clandestin dans les différents camps et d'identifier les différentes formes que cette correspondance illégale a prises. C'est ainsi quà côté des lettres officielles, pourvues d'un faux cachet de censure, nous avons mis en évidence les billets griffonnés à la hâte sur du papier ou du carton, les mots glissés dans des tubes de dentifrice, cousus dans le linge, enroulés dans des cigarettes, cachés dans des ficelles de paquets et sortis illicitement des camps par d'autres moyens encore. Nous avons également établi une liste des psychique réel des détenus et des véritables conditions de vie dans les camps aux consignes données en vue de l'organisation de révoltes et d'évasions en passant par des demandes pressantes afin d'obtenir des médicaments et de la nourriture et la libre expression de sentiments envers des personnes extérieures ou d'un même camp (Kassiber internes) $)^{3}$. Citons également, parmi les thèmes traités, le commentaire de photographies prises àl'insu des gardiens, la justification d’actions de résistance et la défense de l'honneur de compagnons de souffrance ainsi que la séparation évoquée dans les (nombreuses) lettres d'adieu.

Quant aux messages jetés des trains, nous avons montré que ceux qui subsistent témoignent des conditions inhumaines dans lesquelles les déportés devaient voyager et des inquiétudes quant au sort qui leur était réservé; il nous est apparu que, dans bien des cas, ces messages peuvent être assimilés à des lettres d'adieu.

On sait que, parmi les lettres enfouies dans le sol des camps, celles ${ }^{4}$ qui furent dissimulées, en même temps que des chroniques et des journaux intimes, près des crématoires de Birkenau par les membres du Sonderkommando ont connu un grand retentissement. Elles dénoncent en effet avec virulence le génocide perpétré sur les personnes de confession juive et apportent un éclairage saisissant sur la situation morale de ces détenus particuliers, associés malgré eux à ce génocide. Il va de soi que ces lettres font l'objet d'une mention particulière dans notre étude.

Au chapitre consacré aux aspects linguistiques et stylistiques des lettres illégales, nous awons montréqu’elles respectent tantles critères de textualitéqueles principes
(2) Un système sophistiqué,
basé sur des différences de Jambages, a été utilisé par un Buchenwald, Heinrich Adam. à la fin des années 1930. Voir "Letres cryptées d'un résistont communiste allemand interné durant les années 30 dans les camps de concentration nazis n,
Bulletin Trimestriel del la Fondation Auschwitz, $n^{\circ} 93$, octobre-
décembre 2006, p. 85-107. (3) Comme la poignante carte à loccasion de son anniversaire. Le garçon lasssure de son amour mais saccuse aussi du vol de la ration de pain de sa maman
et lui promet de lui restituer de Salmen Gradowski, Herman, rédigées « au coeur de 'enfer , et qui figurent dans louvrage, Des voix sous Sonderkommandos d'A AuschwitzBirkenau, Paris, Calmann-Lévy, 
-Carte clandestine du (Miša) du 19 octobre 1943 adressée à sa maman détenue elle aussi au cam de Theresienstadt régulateurs contrôlant la communication textuelle, ce qui explique les résultats positifs indéniables enregistrés par ces écrits au niveau de la communication. En analysant de plus près les fonctions communicatives dexpression, d'information, d'appe et de contact, nous avons puétablir quelles fonctions prédominaient dans l'ensemble des lettres ainsi que dans chaque catégorie de lettres, de prisonniers et de camps. De même, nous avons mis en évidence les deux pôles thématiques autour desquels les thèmes des lettres s'articulent et analysé l'importance relative de certains thèmes selon les différentes categories de lettres, de prisonniers et de camps. D'un point selon los din de vue en particulier les spécificités de formulation, la langue dans laquelle elles étaient formulées, le niveau de langue utilisé ainsi que les différents styles adoptés par les auteurs. Par ailleurs, nous avons tenté de dégager certains traits relatifs à leurs auteurs, en tenant compte

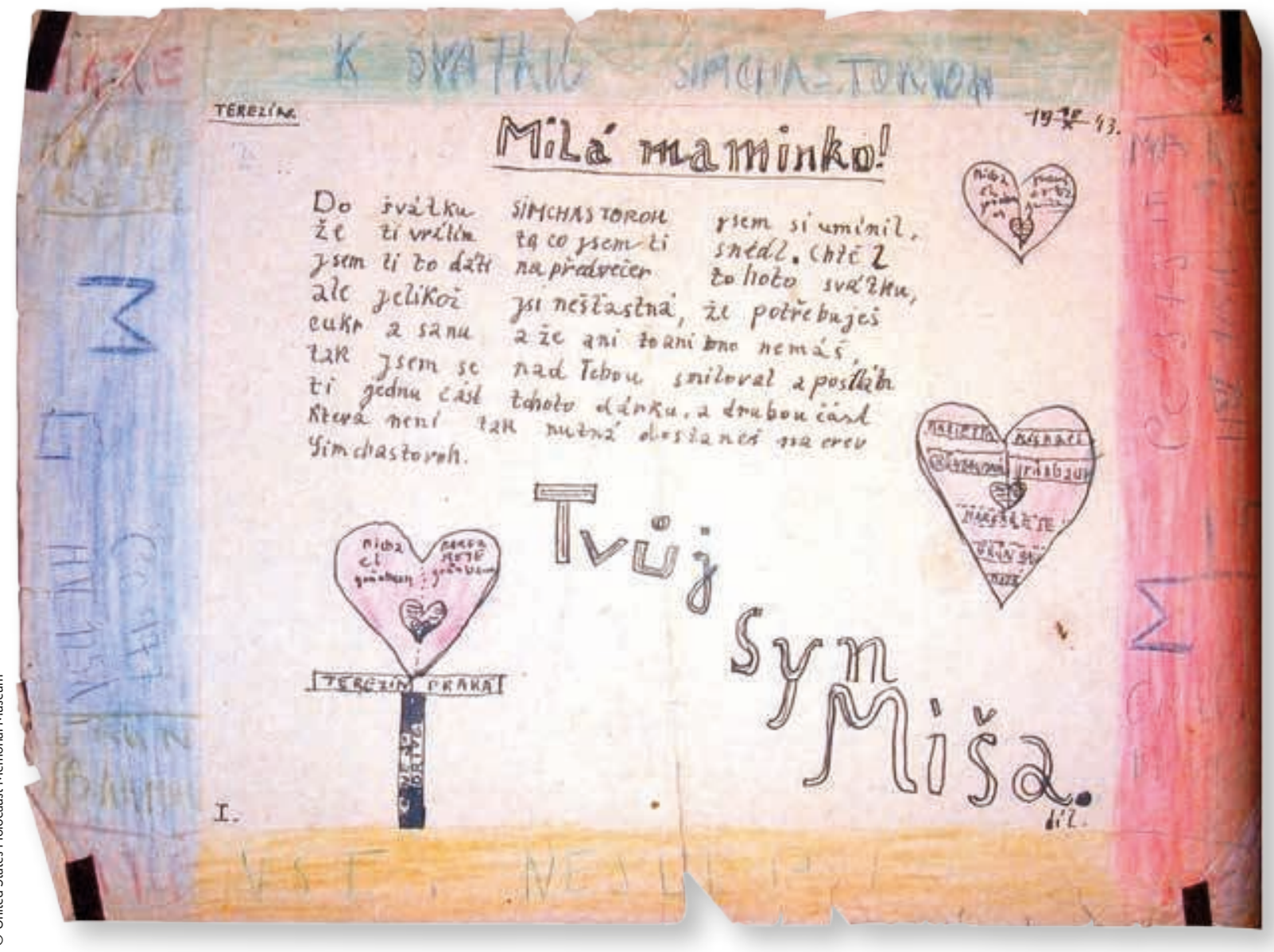

des catégories de prisonniers auxquelles ils appartenaient, de leur statut au sein de la communauté concentrationnaire, de leur pays d'origine et de leur langue. Nous avons ainsi relevé une surreprésentation de détenus de confession juive (forcés de recourir au courrier illégal en raison du fait qu’ils étaient pratiquement exclus du circuit postal officiel) et de résistants polonais (en raison des contacts étroits qu'ils entretenaient avec les réseaux de résistance en Pologne, de l'appui de la population et du fait que leurs lettres ont été relativement bien conservées). De plus, nous avons relevé, parmi les personnes ayant rédigé des messagesillicites, une forte proportion (ly de personnes concernees professionnellement par la correspondance ou l'écriture et/ou ayant exercé dans les camps des fonctions de responsabilité (les « prisonniers
de fonction »).

\section{VALEUR ÉPISTÉMOLOGIQU}

L'étude consacre une place importante à l'examen de la valeur épistémologique des lettres illégales et à la contribution de ces lettres à la recherche historique sur les camps. Hormis le fait que les documents épistolaires constituent en général des témoignages précieux de notre histoire politique, économique, socioculturelle et littéraire, nous avons entrepris d'analyser la valeur des lettres illégales en tant que sources historiques à laide de certains critères tels que la représentativité des auteurs et des thèmes, l'immédiateté et l'authenticité, la fiabilité et la littérarité. auteurs et des thèmes, l'immédiateté et l'authenticité, la fiabilité et la littérarité. Afin d'obtenir des points de comparaison, nous avons appliqué ces mêmes critères
aux autres sources de connaissance, à savoir les actes et documents officiels, les aux autres sources de connaissance, à savoir les actes et documents officiels, les dépositions devantres tribunaux, les récits de témoins oculaires écrits et oraux, les omettre les interviews, les créations littéraires et artistiques, les photographies ainsi que les films documentaires et de fiction.

Au vu des résultats de cette analyse, nous avons pu tirer les conclusions suivantes :

- Étant donné le nombre restreint de lettres illégales émanant des camps, le fait qu’elles n'aient été rédigées que par un nombre réduit de personnes et que ces personnes ne représentent pas toutes les catégories de prisonniers, on ne peut parler d'une grande représentativité dans le chef des auteurs des lettres illégales. Par comparaison, ceux qui ont témoigné oralement et par écrit, laissé des œuvres littéraires et/ou artistiques ou pris en cachette des clichés photographiques ne sont pas, pour différentes raisons, plus représentatifs de l'ensemble des prisonniers. Il en va de même des personnages de romans et des films de fiction, exagérément typés, voire caricaturés. Du point de vue de la représentativité des thèmes, par contre les lettres illég Des couvrent toutesles factes de la vie des prisoniers y conpris les der la vie des prisonnies y 
détenus devant les tribunaux et beaucoup moins fragmentaires que les lettres officielles, qui ne décrivent guère les véritables conditions dans lesquelles évoluaient les prisonniers des camps; elles offrent plus d'éléments d'information que les documents, photographies et procès-verbaux des dépositions des membres de la SS devant les tribunaux militaires. Malgré les moyens techniques sophistiqués engagés, les films documentaires et de fiction s'avèrent également lacunaires, étant donné qu'ils ne représentent que partiellement la réalité et qu'ils renoncentà montrer des scènescommercialement non rentables. Toujours du the point de vue de la repressentativité des thènes, les lettres illégales ne peuven toutefois pas concurrencer la diversité de la poésie et des narrations litteraires, qui sont en mesure de donner une vue plus complète et plus approfondie de la problématique des camps.

Comme elles présentent la double propriété de décrire les conditions de l'époque non pas rétrospectivement, mais en temps réel et sur les lieux mêmes, et de décrire la situation du point de vue des personnes concernées en premier lieu, à savoir les prisonniers, les lettres illégales font partie des sources les plus directes et les plus authentiques dont nous disposons. Elles sont à place sur le même pied que les chroniques des membres du Sonderommando qui sur le furententerés de realisée en cachette dans le camp par les prisonniers, les photos et films documen taires des Alliés (quoique ces derniers aient été retouchés dans nombre de cas) ainsi que les œuvres artistiques qui furent créées dans les camps mêmes (mais qui furent retravaillées la plupart du temps après la détention). Les autres sources sont nettement moins authentiques : les documents et les photos officiels des nazis ne représentent pas la perspective des détenus ; les lettres légales censurées ne reflètent pas l'opinion de leurs auteurs. Les autres " ego-documents », tels que les témoignages, les mémoires, les journaux intimes des anciens prisonniers ainsi que les procès-verbaux des déposition de ceux-ci devant les tribunaux, datent tous de l'époquequi a suivi la période où ils étaient en où ilsectaidevan directes. Ilen va de nênedesfins defiction et des que ceux-ci tentent de créer une illusion d'immédiateté et d'authenticité chez les spectateurs ou les lecteurs.

Les lettres illégales, notamment les lettres clandestines, qu'elles soient de nature privée et destinées aux familles ou plus politiques et transmises à des organisations internationales, possèdent une valeur informative particulièrement élevée et constituent des sources fiables, aussi bien dans leur rôle d'information sur le monde intérieur et létat psychique des prisonniers, qu'en tant que vecteurs de nombrux rensignem teurs den sur lettres illustrées et thématisent des motifs qui semblent éloignés du monde des

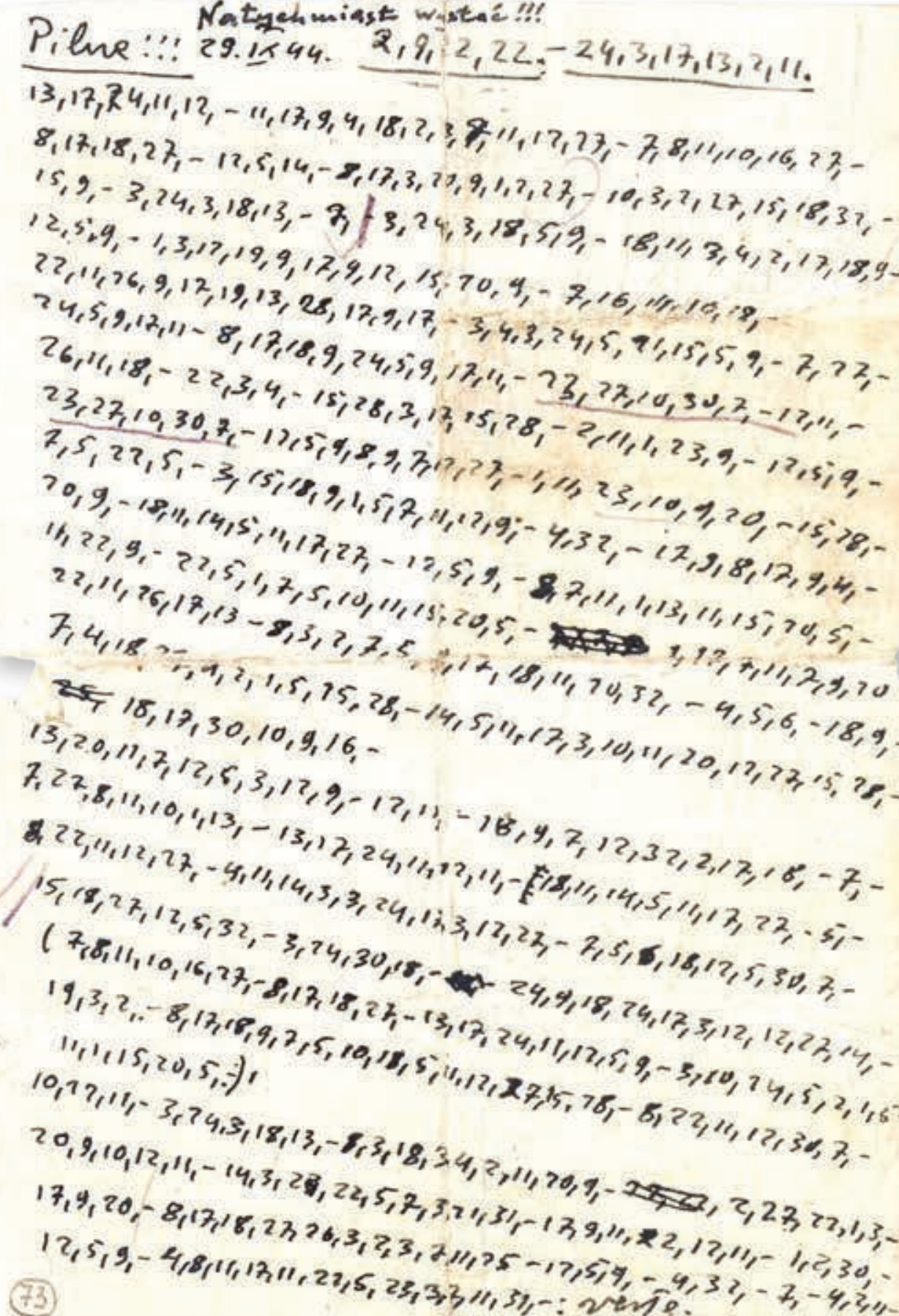

Pilne:", Natyenmiast wastace!?

$13,17,24,11,12,10,13,4,18,2,22,-24,3,17,13,2,11$

$8,17,18,27,-12,5,14,-8,18,3,3,8,11,17,27,-7,8,11,10,16,23,-$

$15,9,-3,24,3,18,13,-8,17,3,27,9,1,2,27,-10,3,2,27,15,18,37-$

$12,5,9,-1,3,12,18,13,-3 / 3,24,3,18,5,9,-18,11,3,4,2,13,3,-$

$22,11,26,9,12,19,9,17,9,12,15,70,4,28,0,3,4,2,1 \geq, 18,9$

$24,5,9,12,12,19,13,28,12,9,17,-3,4,-7,16,11,16,18,-$

$26,11,18,-27,17,18,9,24,5,9,12,11,-23,5,71,15,5,9,-7,27$, $23,27,10,30,7,4,-15,28,3,12,15,28,-2,27,10,30,2,-1 \geqslant, 11,-$

2,5, 22,5, - 3, $12,5,9,8,3,3,12,23,-1,1,2,1,23,9,-12,5 ; 9$.

$10,9,-10,11,14,5,18,9,1,5,2,11,12,9 ;-4,23,10, \geqslant, 20,-15,30$

$11,22,9,-22,5,1112,2 \pi,-12,5,9,-4,32,-i x, 3,8,12,8,2,-$

$22,11,26,12,5,1,2,5,10,11,15,9,-8,2,11,1,13,8,12,9,4,-$

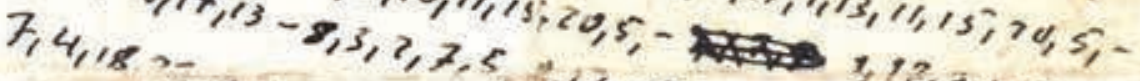

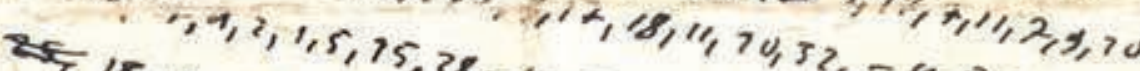

का 18,12,30,10,9, $28,-14,5,11,12,3,10,1120,13,7,6,-18,9$

$7,22,8,11,12,5,3,12,9,-12,11,13,10,11,20,12,73,5,20$

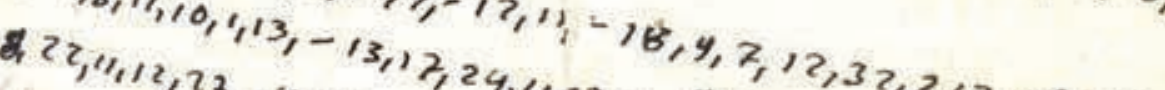

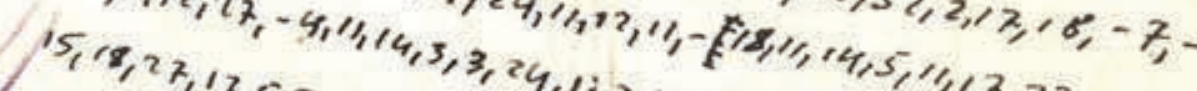

$(7,8,11,12,5,32,-3,24,30,4,3,12,23,-2,5,5,113,23,-5 ;-$

$19,3,2,-8,13,28,17,18,23,-24,18,4,18,24,18,12,5,30,3,-$

$11,1,15,20,5,13,9,2,5,10,18,5,11,24,11,12,5,9,-5,12,12,23,13$,

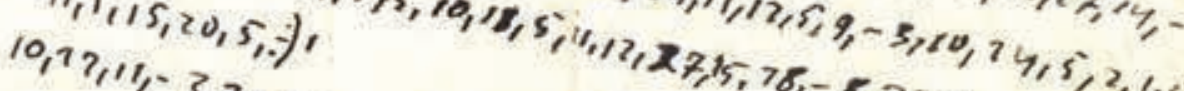

$20,9,10,12,14,3,18,13,-8,3,18,34,2,11,80,8,8,3,23,11,12,30,3,-$

$17,4,20,-8,17,18,23,20,5,2,3,21,31,-13,9,11,20,2,2,23,23,1,3$,

$12,5,9,-4,8,11,1311,20,2,3,2,11,25-12,5,9,2,12,11,-1,2,30$ $2,3,311,3, \ldots-2,1,4,3 \geq-7,-4,3$, (73) 
camps, elles documentent indirectement les véritables sentiments et aspirations des concentrationnaires. Les lettres illégales des camps sont plus fiables que les autres « ego-documents », c'est-à-dire, les témoignages écrits et oraux, les film vidéo, les mémoires et les journaux intimes, qui ontété réalisés après la détention et qui, de ce fait, présentent diverses imperfections, et sont plus proches de la réalité que les déclarations plutôt imprécises des détenus lors des procès. Les lettres illégales sont aussi plus dignes de foi que les actes et documents officiels nazis, rédigés dans la $L$ r nais rejo de lass devantlestibian. et sont plus révelatices que les phographies des SS (qui ne montrent pas les aspectsles plusterribles dela rèalitè). Au même titre queles clichés des Alliés, les clichés réalisés secrètement par les prisonniers ou les réalisations plastiques de ces mêmes prisonniers, les lettres illégales font partie des sources les plus fiables et ont du reste contribué à aider les historiens à propager une représentation plus conforme des camps au sein du grand public. Enfin, elles savèrent, du point de vue de la recherche de la vérité, plus sûres que les films de fiction ou les œuvres littéraires de fiction, qui disposent assez librement des faits historiques et qui, dans les meilleurs des cas, se servent de ces faits comme outils pour exprimer une vérité essentielle, plus générale.

- Au contraire des créations plastiques, cinématographiques ou littéraires d’un certain niveau, les lettres illégales n'ont pas la faculté de transmettre cette vérité essentielle, parce que leur littérarité n'est pas assez développée. Hormis quelques exceptions, elles ne sont pas en mesure de transformer les faits à l'aide de moyens stylistiques élaborés ou de réflexions approfondies, de manière telle à ce que les faits restent ancrés dans la conscience des lecteurs. Il n’empêche que leur extraordinaire authenticité, le fait qu’elles témoignent de la vérité du moment, font qu'elles révèlent, par une autre voie, des fragments importants de la réalité des camps.

En raison de la représentativité de leurs thèmes, de leur authenticité et de leur relative fiabilité et malgré leur valeur littéraire très limitée, les lettres illégales des camps de concentration nazis représentent, en comparaison avec d'autres documents écrits et audio-visuels, des moyens d'investigation tout à fait légitimes dans la recherche sur les camps. Traitées avec le recul critique nécessaire, elles peuvent, en apportant la subjectivité des réflexions et des expériences vécues par les prisonniers, compléter l'objectivité des informations historiques et ains élargir notre champ de vision des événements. Associées aux autres sources de connaissance, elles constituent un ensemble général complexe d'informations dont l'utilisation permet, dans une perspective postmoderne, critique, multiple et interdisciplinaire, d'oboutir à une représentation plus que viaienth et interis comble de la réalité complexe des camps et à une restitution réaliste des sentiments et des
aspirations des concentrationnaires.
En nous appuyant sur notre thèse de la légitimité des lettres illégales en tant que moyens d'investigation, nous avons cru nécessaire de proposer à la fin de notre étude quelques applications pédagogiques des lettres dans le cadre de projets s'inscrivant dans la lutte contre l'oubli. Ces projets, englobant la discussion de problèmes actuels de notre société, utilisent des méthodes d'enseignement basées sur l'autonomie de l'apprenant et visent à l'éducation aux valeurs démocratiques et humanistes (Holocaust Education). $\mathbf{~}$

\section{BIBLIOGRAPHIE}

-Georges Bensoussan, Philippe Mesnard, Carlo Saletti, Des voix sous la
cendre: Manuscrits des Sondert Calmann Lévy, « Mémorial de la Shoah », 2005

- Michael Grunbaum, «L Lettre clandestine du 19 octobre 1943 adressée sa maman n, United States Holocaust Memorial Museum, photo \#2950
(également sous: http://digitalassets ushmmorg photoarchives/detail aspx?:id=1163186\&search=\#29506\&index=1). - Viltor Klemperer, LTI, la langue du Troisième Reich. Carnets d'un
philologue, traduit et annoté par Elisabeth Guillot, Paris, Albin-Michel,
«Bibliotheque Idées », 1996, réédition en livre de poche, Agora Pocket «Biblio
Jacques Presser, Ondergang. De vervolging en verdelging van het
Nederlandse Jodendom 1940-1945, Staatsuitgeverij, 1977.

- Jean-Louis Rouhart, Lettres de lombre. Correspondance illégale dans
les camps de concentration nazis, préfaces de Peter Kuon et de Philippe Mesnard, Liege, Les Territoires de la Mémoire, 2015.

- Jean-Louis Rouhart, «Lettres cryptées dun résistant communiste allemand interné durant les années 30 dans les camps de concentration
nazis, Bulletin Trimestriel de la Fondation Auschwitz $n^{\circ} 93$, octobrenazis », Bulletin Trimestriel
décembre 2006, p. 85-107.
(6) La Lingua Tertii Imperii 